\title{
Discrete dark solitons with multiple holes
}

\author{
Hadi Susanto* \\ Department of Applied Mathematics, University of Twente, P.O. Box 217, 7500 AE Enschede, The Netherlands \\ Magnus Johansson ${ }^{\dagger}$ \\ Department of Physics and Measurement Technology, Linköping University, SE-581 83 Linköping, Sweden \\ (Received 28 July 2004; revised manuscript received 11 February 2005; published 8 July 2005)
}

\begin{abstract}
We consider staggered dark solitons admitted by the discrete nonlinear Schrödinger equation with focusing cubic nonlinearity. In particular, we focus on the study of dark solitons with several holes characterized by the number of zeros in the uncoupled case. Such structures reveal interesting behaviors, such as stable intersite dark solitons. All of the structures have no counterpart in the strong coupling limit since they disappear in a saddle-node bifurcation. We also consider the evolution of structures with multiple holes representing an interaction between multiple dark solitons in a very discrete case.
\end{abstract}

DOI: 10.1103/PhysRevE.72.016605

PACS number(s): 42.65.Tg, 05.45.Yv, 63.20.Pw

\section{INTRODUCTION}

Nonlinear localized modes in discrete systems exist due to the interplay between lattice coupling and nonlinearity effects. One of the most studied nonlinear lattice models is the discrete nonlinear Schrödinger equation [1], which also admits such spatially localized modes or discrete solitons. Several diverse areas where discrete solitons appear in applications, such as biophysics, nonlinear optics, solid state physics, waveguide arrays, and Bose-Einstein condensates in optical lattices, are described in [2]. Many of these systems can, in some limit, be described by models related to the discrete nonlinear Schrödinger equation, and in particular, the latter two have received considerable attention in recent years.

The first discussion on discrete spatial solitons in a periodic optical structure created by using an array of optical waveguides was introduced by Christodoulides and Joseph [3]. Since then, discrete spatial solitons were studied intensively. Extensive reviews of discrete solitons in waveguide arrays with their applications can be read in $[4,5]$. Recently, it has been shown that discrete solitons can be observed and manipulated experimentally [6-10]. Eisenberg et al. [6] reported the first experimental observation of discrete spatial solitons in gallium arsenide AlGaAs waveguide arrays.

The use of discrete nonlinear Schrödinger models to describe Bose-Einstein condensates trapped in periodic optical lattices was suggested by Trombettoni and Smerzi [11], and recent experiments [12] also confirm the existence of selflocalized discrete solitons as predicted by these models.

In addition to localized "bright" solitons, it is well known that also dark solitons, consisting of a localized dip in a homogeneous background intensity, may generally exist. Theoretical reviews of dark solitons and their applications in nonlinear optics can be read in, e.g., $[13,14]$. In homogeneous continuum systems, bright and dark solitons normally

\footnotetext{
*Electronic address: h.susanto@math.utwente.nl

†Electronic address: mjn@ifm.liu.se
}

do not appear simultaneously, since the former generally require an effectively focusing and the latter an effectively defocusing nonlinearity. Thus, for example, without any periodic potential dark solitons are observed in Bose-Einstein condensates with repulsive interatomic interaction [15], and bright solitons when the effective interactions are attractive [16].

In the present work, we focus on the study of discrete dark solitons. It is a fundamental property of discrete systems (or, more generally, spatially periodic systems with a band-gap structure for the dispersion relation of linear waves), that bright and dark localized modes may appear for the same physical system. Thus, for example, waveguide arrays with the same material system can be used to form both bright solitons and dark solitons, since the waveguide diffraction properties can be modified [7,10]. In [7,10], Morandotti and co-workers reported experimental observations of discrete dark solitons in a waveguide array. Since in these cases the nonlinearity itself is focusing, the discrete dark solitons become "staggered," i.e., the constant-intensity background has opposite phases at neighboring sites (potential wells). Analogously, when a Bose-Einstein condensate with repulsive interatomic interactions is subjected to a periodic optical potential, bright discrete (gap) solitons with staggered tails are observed [12].

There are many references on the study of discrete dark solitons (see, e.g., [17-20]). Johansson and Kivshar [19] discussed the existence and instabilities of two families of fundamental dark solitons in the discrete nonlinear Schrödinger equation, which were shown to exist along a continuous path ranging from the highly discrete limit of uncoupled lattice sites, to the continuous limit. In particular, it was found that oscillatory instabilities were induced by a weak inherent discreteness. Here, we specifically discuss the existence and stability of more complicated types of strongly discrete dark solitons with more than one hole or notch in their structures. Such modes should be of particular interest for experimental realizations with waveguide arrays, where one may quite carefully control the generated output by tuning the initial beams. In particular, it is of interest from a theoretical as well as a practical viewpoint to know whether such nonfunda- 
mental dark solitons may be stable even when the fundamental dark modes are unstable. Although, in some sense, our work has a spirit similar to earlier work on multiple localized bright modes (see, e.g., [21,22], and a recent mathematical work [23] and references therein), evidently, the physical context of discrete dark solitons with multiple holes is quite different.

The present work is organized as follows. We describe the discrete equation considered in this work and the two basic modes admitted by it in Sec. II. In that section, we also recapitulate the calculation of the oscillatory instability of the modes derived in Ref. [19], using, however, a different parametrization that will also be employed for the remaining parts of this study. In Sec. III, we discuss composite dark solitons characterized by the property of having several consecutive sites with zero intensity at the uncoupled limit. We show that there are two different types of configurations identified by the signs of the nonzero field just nearby the zeros. Even though numerics shows that both configurations are stable in the weak-coupling regime for large but finite systems, we conjecture that only one of the two configurations can be stable for infinite systems. In Sec. IV, we present very discrete structures resembling multiple dark solitons. We close the paper with a conclusion in Sec. V.

\section{THE MATHEMATICAL PROBLEM AND ITS BASIC MODES}

The discrete nonlinear Schrödinger equation we consider in this work is given by $[1,19]$

$$
i \dot{\phi}_{n}+C\left(\phi_{n+1}+\phi_{n-1}\right)+\left|\phi_{n}\right|^{2} \phi_{n}=0,
$$

where, in the context of optical waveguides, $\phi_{n}$ is the complex envelope of the electric field at the $n$th site or waveguide and $C$ is the coupling constant between the adjacent sites. The dot generally stands for the derivative in time, although for spatial solitons in waveguide arrays it physically represents a derivative with respect to the longitudinal spatial coordinate. Without loss of generality, we choose $C$ $>0$.

Equation (1) has exact stationary solutions of the form

$$
\phi_{n}=\psi_{n} e^{i \Lambda t} .
$$

Let $\psi_{\infty}=\lim _{n \rightarrow \pm \infty}\left|\phi_{n}\right|$. Because we are considering dark solitons admitted by Eq. (1), then the boundary conditions $\psi_{\infty}$ are nonvanishing and constant. Generally, for a background wave vector $k$, we may assume $\lim _{n \rightarrow+\infty} \psi_{n}=\psi_{\infty} e^{i k n}$ and $\lim _{n \rightarrow-\infty} \psi_{n}=\psi_{\infty} e^{i k n} e^{-i \delta}$, where $\delta$ is the total phase shift associated with the dark soliton. The relation between the boundary condition and the frequency $\Lambda$ is given by $\left(\psi_{\infty}\right)^{2}=\Lambda$ $-2 C \cos k[17,19]$. To obtain a stable solution, we must first require the background wave to be modulationally stable, which is obtained when $C \cos k<0$ [24].

A special case considered in this paper is when $k=\pi$, which corresponds to the so-called staggered transformation, i.e., $\phi_{n} \rightarrow(-1)^{n} \phi_{n}$, relating Eq. (1) with $C>0$ (nonlinearity and coupling of same sign) to the same equation with $C$ $<0$ (nonlinearity and coupling of opposite sign). A staggered dark soliton is one solution admitted by Eq. (1) with $C>0$, thus corresponding to an unstaggered dark soliton for $C<0$. As discussed in Sec. I, experiments on the creation and observation of staggered dark solitons in waveguide arrays have been recently reported $[7,10]$. A theoretical study of the more general case with nonzero $k \neq \pi$ has also recently been reported [25]. In this case, the fundamental stationary dark solitons were found to be "gray" with nonzero minimum intensities.

There are two fundamental types of staggered stationary dark solitons, i.e., the on-site mode ( $A$ mode) and the intersite mode ( $B$ mode), which are stable and unstable, respectively, for small $C$ [19]. At $C=0$ and $\Lambda=1$, the $A$ mode takes the form

$$
\psi_{n}=(\ldots,-1,+1,0,-1,+1, \ldots),
$$

described by a single "hole" inserted in a constant amplitude wave background. The $B$ mode is described by

$$
\psi_{n}=(\ldots,-1,+1,-1,-1,+1,-1, \ldots),
$$

and can be obtained by removing one site from the constantamplitude $k=\pi$ background. In both cases, an additional total phase shift $\delta=\pi$ appears across the defect. From now on, to characterize a particular solution, we use the corresponding solution at $C=0$.

We call the two above modes the basic modes since they both can be followed uniquely from the case $C=0$ to $C$ $\rightarrow \infty$ [19]. If the continuation, as in [19], is done by varying the frequency as $\Lambda=1-2 C$ to keep a constant background intensity $\psi_{\infty}=1$ (alternatively, continuation of the $A$ mode (3) may also be performed at constant complementary norm as defined, e.g., in [14]), the latter is a continuum limit where the standard nonlinear Schrödinger dark-soliton solution (e.g., [14]) is obtained. In [19], the stability of the $A$ mode was discussed, as well as a critical value of the coupling parameter $C_{c r}$ above which the mode becomes unstable identified. At the critical value, one pair of discrete eigenvalues of the solution collides with eigenvalues coming from the continuous spectrum, and results in four complex eigenvalues with nonzero real part for $C>C_{c r}$. Such an instability is called oscillatory instability.

In this section, we will reproduce some of the calculations in [19], using, however, for illustrative purposes, a different parametrization. Thus, we here keep $\Lambda=1$ fixed and let $\psi_{\infty}$ vary as a function of $C$, contrary to [19]. Effectively, this corresponds to rescaling also the coupling constants from [19] (denoted as $C^{[19]}$ ) as $C=C^{[19]} /\left(1-2 C^{[19]}\right)$. Note that there is one-one correspondence $0<C<\infty \leftrightarrow 0<C^{[19]}<\frac{1}{2}$, but that the regime $C^{[19]}>\frac{1}{2}$ (and thus the continuum limit), cannot be obtained by smooth continuation increasing $C$ at fixed $\Lambda$. Moreover, the intensity of the background wave $\left(\psi_{\infty}\right)^{2}=1+2 C$ diverges when $C \rightarrow \infty$. However, this does not matter for the studies of dark solitons with multiple holes in the forthcoming sections, since these exist only for relatively small values of the coupling $C$.

We look for a dark soliton of Eq. (1) from a given initial condition at $C=0$ using the Newton-Raphson continuation method with periodic boundary conditions (see, e.g., [26]). In general, if nothing else is stated, we use the number of 


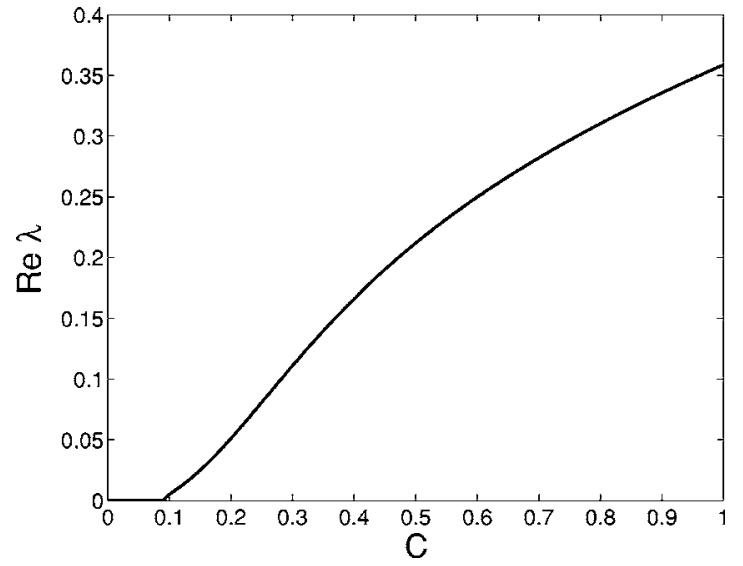

FIG. 1. Real part of the unstable eigenvalues of an on-site dark soliton of Eq. (1) as a function of the coupling parameter $C$.

sites $N=201$ and $\Lambda=1$. To assure that the obtained results are not influenced by the finite number of sites, we compare them with the calculation using a higher number of sites.

The linearization of (1) around an exact solution $\left\{\psi_{n}\right\}$ yields, by writing $\phi_{n}(t)=\left[\psi_{n}+\epsilon_{n}(t)\right] e^{i \Lambda t}$,

$$
i \dot{\epsilon}_{n}-\Lambda \epsilon_{n}+C\left(\epsilon_{n+1}+\epsilon_{n-1}\right)+2\left|\psi_{n}\right|^{2} \epsilon_{n}+\psi_{n}^{2} \bar{\epsilon}_{n}=0,
$$

with the overbar representing the complex conjugation. This leads to a standard linear eigenvalue problem (see, e.g., [19] and references therein). Let us denote an eigenvalue of the considered solution with $\lambda$. The spectrum $\lambda$ generally may consist of discrete spectrum (eigenvalues) and continuous spectrum. The continuous spectrum can be obtained by calculating the dispersion relation of a linear wave

$$
\epsilon_{n}=a e^{i(\kappa n-\omega t)}+b e^{-i(\kappa n-\omega t)},
$$

satisfying Eq. (5) with $\psi_{n}=(-1)^{n} \sqrt{\Lambda+2 C}$. With $\lambda=i \omega$, the continuous spectrum is obtained from the dispersion relation to lie in the interval $\left(0, \pm i \sqrt{32 C^{2}+8 \Lambda C}\right)$.

When $C \rightarrow 0$, the entire continuous spectrum of the $A$ mode tends to zero plus an eigenvalue at $\pm i \Lambda$ due to the presence of the hole. For nonzero $C$, we use MATLAB to solve the above eigenvalue problem for given $\left\{\psi_{n}\right\}$. The unstable eigenvalue of the $A$ mode shown by the maximum of $\operatorname{Re}(\lambda)$ as a function of $C$ is presented in Fig. 1. Our numerical scheme shows that the instability is getting large as the coupling parameter $C$ increases. The critical coupling parameter $C_{c r}$, above which the staggered dark soliton becomes unstable, is found at $C_{c r} \approx 0.0902$. Note that $C_{c r}^{[19]}=C_{c r} /(1$ $\left.+2 C_{c r}\right) \approx 0.0764$. Note also that the rescaling of background intensity also rescales the stability eigenvalues $\lambda$ compared to the corresponding eigenvalues $\lambda^{[19]}$, as $\lambda=\lambda^{[19]} /(1$ $-2 C^{[19]}$ ). As a consequence, $\lambda \rightarrow \infty$ as $C \rightarrow \infty$, and the maximum of the instability at $C^{[19]} \approx 0.32$ does not appear with the parametrization used in the present work.

The above result can be approximated analytically. Note that the phonon band lies between 0 and $\pm i \sqrt{32 C^{2}+8 \Lambda C}$. For small but nonzero $C$, the eigenvalues at $\pm i \Lambda$ move towards 0 along the imaginary axis. At some value of $C$ there will be a collision between these eigenvalues and the edges of the con- tinuous spectrum, creating two pairs of complex eigenvalues but with nonzero real part (oscillatory instability) [19]. Assuming that, for small $C$, the eigenfunction is strongly localized at the hole, then its oscillation frequency $\omega=-i \lambda$ is close to the dark soliton frequency $\Lambda$. Therefore, we obtain

$$
C_{c r} \approx \frac{\sqrt{3}-1}{8} \Lambda \approx 0.09151 \Lambda \text {. }
$$

Contrary to the $A$ mode, the $B$ mode, which is an intersite dark soliton, is always unstable for any values of positive $C$ [17-19].

\section{DARK SOLITONS WITH CONSECUTIVE HOLES}

In this section, we will discuss the stability of dark soliton structures with consecutive holes characterized by the number of zeros in the solution for $C=0$. Again, there are two possible types of modes: the $A$ modes and the $B$ modes. The names are from the basic modes to which we add some zeros in the middle of the solution. As before, we fix the value of $\Lambda$ at 1 .

\section{A. The $A$ modes}

In this subsection, we will focus on the first mode, i.e., the $A$ mode. The $A$ mode with two zeros is simply the basic $A$ mode given by Eq. (3) with two zeros instead of one. Hence, the $A$ mode with two holes is given by (at $C=0$ )

$$
\psi_{n}=(\ldots,-1,+1,0,0,-1,+1, \ldots) .
$$

A sketch of the solution for $C>0$ using the above initial condition is shown in Fig. 2(a). Looking at the magnitude value $\left|\psi_{n}\right|^{2}$ of this structure, we can say that this solution also represents an inter-site dark soliton as is the basic $B$ mode Eq. (4). However, there is a fundamental difference: since each inserted hole also contributes a phase shift of $\pi$, the total phase shift associated with the two-hole $A$ mode is $\delta$ $=2 \pi$, while that of the basic $B$ mode is $\pi$. Thus, these two solutions correspond to different boundary conditions. In our case, using periodic boundary conditions for the background $k=\pi$ wave, a phase shift $\delta=\pi$ requires the number of sites $N$ to be odd, while $\delta=2 \pi$ requires even $N$. These observations also allow us to interpret the two-hole $A$ mode as a tightly bound state of two basic $A$-mode dark solitons.

An interesting result is that this intersite dark soliton is stable for some values of the coupling parameter $C$. Hence, we have found a stable intersite dark soliton. The real part of the most unstable eigenvalues of the $A$ mode with two holes as a function of $C$ is shown as a solid line in Fig. 2(b). Note that there is an analogy with the so called "twisted localized modes" [27], which are intersite "bright" phase-twisted solitons, $\psi_{n}=(\ldots, 0,0,+1,-1,0,0, \ldots)$ at $C=0$, also stable for small $C$ [28].

In Fig. 2(c), we show the evolution of a dark soliton with two holes at $C=0.2$. We use a fourth-order Runge-Kutta scheme to do the integration in time of Eq. (1). As an initial condition, we use a numerically obtained dark soliton perturbed by a random function with maximum value $10^{-4}$. It is clearly seen from the inset of Fig. 2(c) that after some time, 

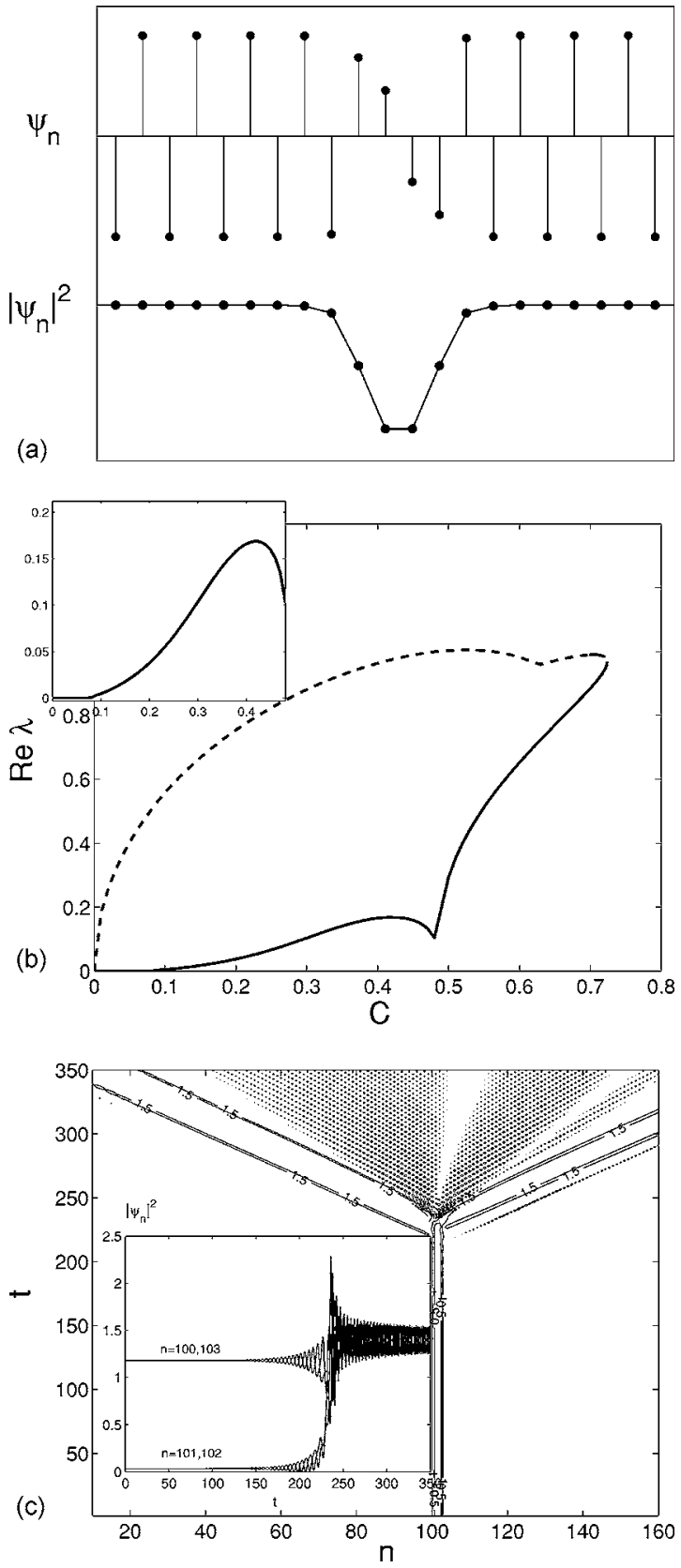

FIG. 2. (a) The oscillation amplitude of the phase field $\psi_{n}$ at each site and the value $\left|\psi_{n}\right|^{2}$ of an intersite dark soliton. The two holes move with out-of-phase configuration. (b) Real part of the unstable eigenvalues of the solution as a function of the coupling parameter $C$. The inset enlarges the lower branch where it becomes clear that the intersite-like dark soliton is stable for small $C$. (c) The evolution of an $A$ mode soliton at $C=0.2$. In the contour plot, lines correspond to intensities $\left|\psi_{n}\right|^{2}=0.5,1$ and 1.5. The inset shows the detailed dynamics of a few sites at and around the holes. One can see that the holes also move in time in out-of-phase configuration.

the holes transfer energy to the neighboring sites, so that all the sites start oscillating. By studying more carefully the dynamics shown in the contour plot, one may identify outgoing lines with small-amplitude traveling gray solitons, with small intensity dips associated with localized phase shifts, which survive for intermediate time ranges.
The value of the critical coupling parameter $C_{c r}$, above which the state is unstable, is found numerically at 0.0782 . This value can be approximated analytically by doing the same kind of calculation as given in the previous section.

Let the holes be at the sites number $n=1$ and $n=2$. By assuming that the eigenfunction is completely localized at the holes, then $\epsilon_{n}=0$ for $n \neq 1,2$. Hence, we obtain a system of two coupled equations for $\epsilon_{1}$ and $\epsilon_{2}$. We know that, for the eigenmode causing the instability, the holes are oscillating with out-of-phase configuration, from which we can assume that $\epsilon_{1}=-\epsilon_{2}$. Since $\psi_{\{1,2\}}=0$, we obtain that the holes oscillate with frequency $\Lambda-C$. Equating this frequency with the edge of the continuous spectrum gives an approximate critical value of the coupling parameter, i.e.,

$$
C_{c r}=\frac{2 \sqrt{14}-5}{31} \Lambda \approx 0.0801 \Lambda
$$

which agrees quite well with the numerical result.

If we keep increasing the coupling $C$, at a particular value the Newton-Raphson will fail and the initial solution will jump to another solution. This is a typical scenario of the disappearance of a solution in a saddle-node bifurcation. For our $A$ mode with two holes, the bifurcation point $C_{b}$ is at $C_{b} \approx 0.7237$.

Our analysis reveals that the $A$ mode with two holes collides in a saddle-node bifurcation with a solution coded by $\psi_{n}=(\ldots,-1,+1,+1,-1,-1,+1, \ldots)$. The stability of the latter solution is shown as a dashed line in Fig. 2(b) (note that it is always unstable).

If we look at the stability curves in Fig. 2(b) then we will notice that at some points, the curves form a cusp. The points correspond to a structural change of the most unstable eigenvalues. The cusp point in the lower branch at $C \approx 0.48$, for instance, is the point at which the most unstable and complex eigenvalues become purely real.

We have done also the calculation on the stability and existence of the $A$ mode with multiple consecutive holes. The result is summarized in Fig. 3. It is clear that the existence region is decreasing as the number of zeros increases, while the critical coupling parameter $C_{c r}$ is almost independent of the number of zeros. One important difference between dark solitons with even and odd number of holes of this mode is that the former one has no $\pi$ phase shift of its background field across the holes (or, equivalently, that the total phase shift $\delta$ is an even/odd multiple of $\pi$, respectively).

\section{B. The $B$ modes}

After considering an expansion of the basic $A$ mode by adding a number of zeros in the solution, one may question also what happens if we put zeros in the center of the basic $B$ mode. It is known that the basic $B$ mode is unstable [17-19].

We start with considering only one zero in the middle of a dark soliton such that the structure in the uncoupled case is given by $(\ldots,-1,+1,0,+1,-1, \ldots)$. Note that the total phase shift for this solution is $\delta=0(\bmod 2 \pi)$. Using $N=202$, we found only a very small stable region for this structure, which becomes unstable for $C>C_{c r} \approx 0.0050$. The instability 


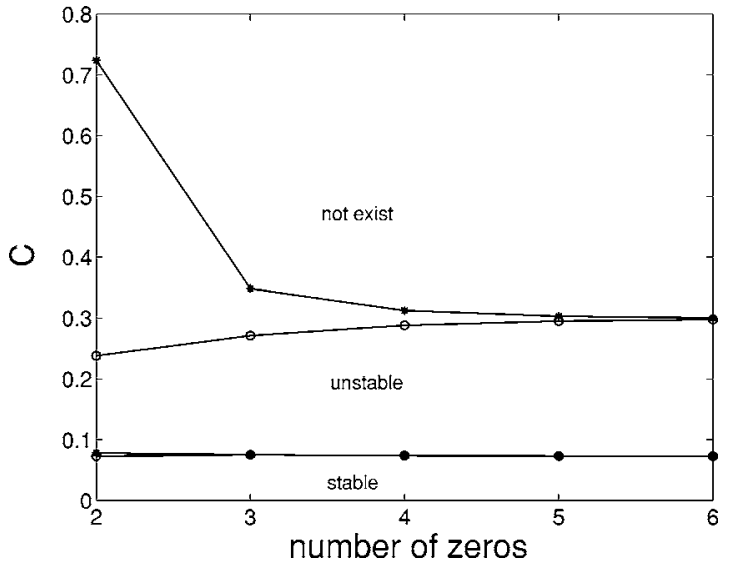

FIG. 3. A diagram of the existence and the stability region of dark solitons with multiple zeros in the $A$-mode configuration (-*-) and the $B$-mode configuration (-O-). The calculations for the $A$ modes $[B$ modes] are done with $N=201$ or $N=202$ for an odd [even] or even [odd] number of zeros, respectively.

occurs through an eigenvalue collision at 0 , and thus the resulting unstable eigenvalues are purely real. It can be seen to correspond to interactions between the two "+1"-sites on either side of the hole. For larger coupling, $C \approx 0.081$, also an oscillatory instability with complex eigenvalues sets in, corresponding to an interaction between a mode localized at the hole and the continuous spectrum, as described above for the $A$ modes. At some value of the coupling, $C=C_{b}$ $\approx 0.1834$, the structure also disappears in a saddle-node bifurcation with a solution coded by $(\ldots,-1,+1,+1,+1$, $-1, \ldots)$.

Next, we consider when there are two zeros in the $B$ mode, yielding $\delta=\pi(\bmod 2 \pi)$. A sketch of the solution at finite $C$ is given in Fig. 4(a). It is interesting to note that the structure is stable for small $C$, in a non-negligible regime for $N=201$. The stability curve is shown in Fig. 4(b) as a solid line. The numerically obtained critical coupling parameter is $C_{c r}=0.0728$, where the real instability sets in, analogously to the case for the one-hole $B$ mode. At a slightly larger value of $C$ also the complex instability appears as above, but the dominating eigenvalue is always the real one. This solution will also disappear in a saddle-node bifurcation at $C_{b}$ $=0.2381$. One can guess already that this $B$-mode soliton collides with the solution coded by $(\ldots,-1,+1,+1,+1$, $+1,-1 \ldots)$. The stability of the latter solution is shown in dashed-line in Fig. 4(b). The evolution of a dark soliton of this mode for $C=0.2$ is shown in Fig. 4(c). In Fig. 3 we show the existence and the stability region of the $B$ mode with multiple zeros. Please note that the above numerical result is obtained by using the total number of sites $N=201$ [even number of zeros, $\delta=\pi(\bmod 2 \pi)]$ or $N=202$ [odd number of zeros, $\delta=0(\bmod 2 \pi)]$.

We test the numerical result with a larger number of sites. Another interesting thing is that the critical value $C_{c r}$ is $d e$ creasing as a function of the total number of sites. For the $B$ mode with two zeros we obtained that using $N=301, C_{c r}$ $\approx 0.0592$. Using $N=401$, we obtained $C_{c r} \approx 0.0511$. From this we conclude that the stabilization we have here is pre-
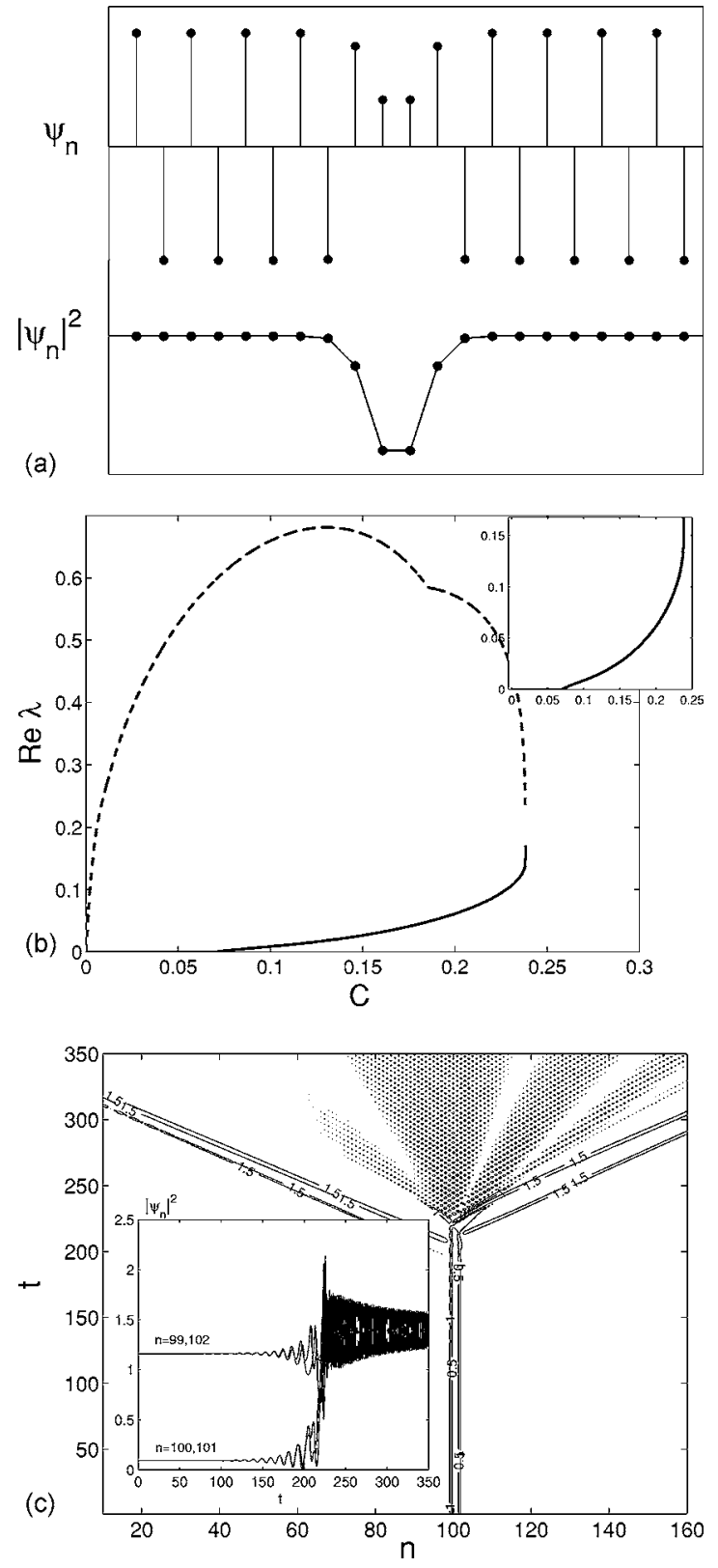

FIG. 4. The same as Fig. 2, but the two holes are in the $B$ mode configuration.

sumably due to the finite number of sites and the boundary conditions. In the limit of $N \rightarrow \infty$, a $B$-mode dark soliton with multiple zeros seemingly has $C_{c r}=0$. Analogously, the stability region for the $B$ mode with one hole increases for smaller $N$, so that, e.g., $C_{c r} \approx 0.011$ for $N=100$ and $C_{c r} \approx 0.018$ for $N=60$ for this mode.

Nevertheless, taking a different value for the number of sites does not change the boundary of the existence of this mode. Hence, the number of sites, as long as it is large enough, will not influence the value of $C_{b}$. It can be simply understood since $C_{b}$ is determined by the interaction of the holes with only some neighboring sites. The same is true for the location of the oscillatory instability threshold, which is also roughly independent on the system size. As a consequence, when the number of sites is decreased for a given 

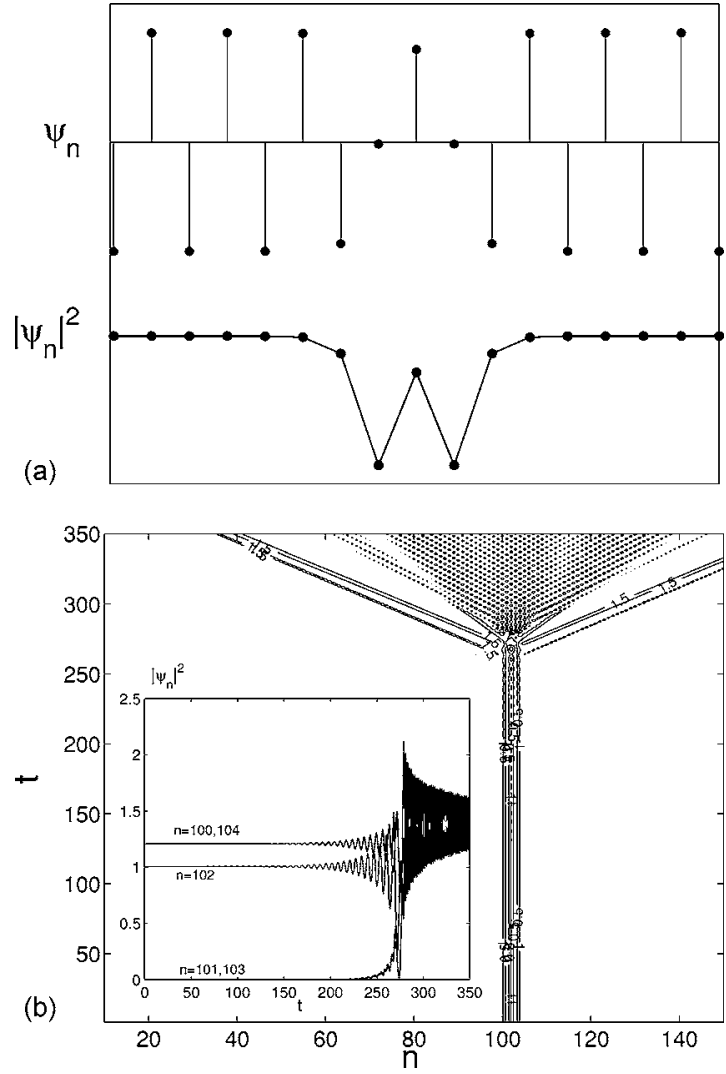

FIG. 5. (a) A solution representing two dark solitons. The two solitons are only separated by one lattice site. (b) The evolution of a solution given in (a) at $C=0.2$. The inset shows the evolution of several sites at and around the holes.

mode, the first encountered instability determining $C_{c r}$ may change from being real to oscillatory. Thus, for example, for the $B$ mode with two holes, the oscillatory instability appears for smaller $C$ than the real one for $N<176$.

\section{STRUCTURES RESEMBLING INTERACTION OF MULTIPLE DARK SOLITONS}

Besides structures with holes placed consecutively in an array, we can consider also the case of staggered dark solitons with several holes but separated by several nonzero $\phi_{n}$. Such structures can be considered as multiple dark solitons. The interaction of the solitons can be seen through the stability of the structure compared to the solution representing one soliton. Unfortunately, a single soliton already becomes unstable for very small coupling $C$ which makes it difficult to see the influence of the presence of one soliton to the other.

As an example, we consider a structure that can be thought as a representation of two dark solitons. The simplest one is given by $(\ldots,+1,-1,0,+1,0,-1,+1, \ldots)$. A sketch of this solution at finite $C$ is presented in Fig. 5(a).

We have analyzed the stability of this solution. We obtained numerically that $C_{c r} \approx 0.0908$. Note that the critical coupling is slightly higher than the $C_{c r}$ of one soliton. We can say that the two solitons are stabilizing each other.

In Fig. 5(b) we present the evolution of a two-dark-soliton structure at $C=0.2$. We can see that after a while, the nonzero field in the middle of the two zeros becomes oscillating which is followed by a collision between the two holes. The final state is that all the sites oscillate.

We have also considered interaction of many holes given by the code $(\ldots,-1,+1,0,-1, \ldots, 0,+1,-1, \ldots)$. Up to four digit decimals, all the structures lose their stability at $C_{c r}$ $\approx 0.0908$.

Besides the solution shown here, one can make other solutions representing two dark solitons as a function of, e.g., number of sites between the two zeros. Yet, we believe that all such solutions will also disappear in a bifurcation with other structures.

\section{CONCLUSIONS}

To conclude, we have discussed the stability and the existence of staggered discrete dark solitons with multiple holes in the focusing discrete nonlinear Schrödinger equation. It is shown that all of the considered structures disappear in a saddle-node bifurcation. Even so, an important observation from our numerical investigations is that all such structures indeed are stable for a range of small values of the coupling parameter, when considered for finite lattices with periodic boundary conditions. For $A$-mode solitons, characterized by antiphase oscillations of the two sites surrounding the holes, the results are not sensitive to system size effects, and thus the stability regime should survive also in the limit of an infinite system. In particular, for weak coupling the $A$ mode with two holes is a stable intersite dark soliton, which, in analogy to the well-known "twisted localized modes" [27] may be termed a "twisted dark mode," accounting for the fact that it is associated with a total phase shift $\delta=2 \pi$, while $\delta=\pi$ for the fundamental dark solitons. However, for $B$-mode solitons, having in-phase oscillations at the sites neighboring the holes, the stability regime shrinks when the system size is increased, seemingly disappearing asymptotically as $N \rightarrow \infty$.

For all the studied dark modes with two or more consecutive central holes, we found that the stability regime is smaller than that of the earlier studied [19] basic A mode (with one hole). We have also considered structures representing multiple dark solitons, with nonconsecutive holes. Especially for the case of two solitons (holes) separated by one lattice site, we have shown that the solitons stabilize each other so that the oscillatory instability happens at a coupling parameter value slightly higher than for a single A-mode soliton.

Let us finally relate our results to what is known generally from earlier and recent rigorous work on linear stability of multisite solitons in the (focusing) discrete nonlinear Schrödinger equation. Using Aubry's theory of effective action [29], it was proven [28] that two-site antiphased solutions are stable for weak coupling, if they are placed at neighboring sites (twisted localized mode) or have one hole in between. Similarly, it was proven in [19] that the staggered basic $A$-mode dark soliton is also stable for small $C$. 
This led to the conjecture, that generally structures composed by antiphased excited sites, separated by an arbitrary number of hole sites, should be stable for weak coupling, while each pair of in-phase excited sites (neighboring or separated by empty sites) should give rise to one pair of unstable (real) eigenvalues. Very recently, this was proven rigorously under quite general conditions (Theorem 3.6 in [23]). However, since [23] exclusively deals with localized modes (with finite number of excited sites) in infinite systems, the proof does not cover the dark modes considered by us. Still, we note that the stability of the $A$ modes for small $C$ is in agreement with the conjecture.

However, the fact that we found the $B$ modes with two in-phase excited sites, separated by holes, also to be stable for small $C$ for any finite $N$ shows, that the conjecture is generally not true for finite systems. Heuristically, we might explain this as follows: even though the dynamics locally would like to twist the central in-phase oscillators towards the favorable antiphase configuration, this cannot be done globally for a finite system with periodic boundary conditions, without creating a phase mismatch at the boundary. Thus, the solution remains stable as long as the "gain" from twisting the central part is smaller than the "loss" resulting from twisting the rest of the mode. Since, perturbatively for small $C$ the phase interaction between oscillators spaced $m$ sites scales as $C^{m}$ [28], the former scales as $C^{p+1}$ for a $B$ mode with $p$ holes (and is independent of $N$ ), while the latter plausibly (for homogeneous phase torsion) scales as $C / N$ (and thus decreases with $N$ ). Consequently, the stabilizing part from the rest of the lattice will dominate the destabilizing contribution from the central sites for small $C$ and $N$ if $p \geqslant 1$, while the destabilizing central part takes over as $C$ and/or $N$ increases. This argument thus predicts the (real) instability threshold for these modes to scale as $C_{c r}$ $\sim(1 / N)^{1 / p}$, which agrees well with the numerical results reported in Sec. III B.

\section{ACKNOWLEDGMENTS}

H.S. thanks P.G. Kevrekidis for fruitful discussions and S.A. van Gils for being a supportive supervisor. M.J. thanks Yu. S. Kivshar for initiating his interest in the topic. This work is supported by the Royal Netherlands Academy of Arts and Sciences (KNAW) (H.S.), and the Swedish Research Council (M.J.).
[1] J. C. Eilbeck and M. Johansson, in Localization and Energy Transfer in Nonlinear Systems, Proceedings of the Third Conference, San Lorenzo de El Escorial Madrid, edited by L. Vázquez, R. S. MacKay, and M. P. Zorzano (World Scientific, Singapore, 2003), p. 44; e-print nlin.PS/0211049.

[2] D. K. Campbell, S. Flach, and Yu. S. Kivshar, Phys. Today 57(1), 43 (2004).

[3] D. N. Christodoulides and R. I. Joseph, Opt. Lett. 13, 794 (1988)

[4] A. A. Sukhorukov, Yu. S. Kivshar, H. S. Eisenberg, and Y. Silberberg, IEEE J. Quantum Electron. 39, 31 (2003).

[5] Yu. S. Kivshar and G. P. Agrawal, Optical solitons: From Fibers to Photonic Crystals (Academic, Amsterdam, 2003).

[6] H. S. Eisenberg, Y. Silberberg, R. Morandotti, A. R. Boyd, and J. S. Aitchison, Phys. Rev. Lett. 81, 3383 (1998).

[7] R. Morandotti, H. S. Eisenberg, Y. Silberberg, M. Sorel, and J. S. Aitchison, Phys. Rev. Lett. 86, 3296 (2001).

[8] F. Lederer and Y. Silberberg, Opt. Photonics News 13(2), 48 (2002).

[9] D. N. Christodoulides, F. Lederer, and Y. Silberberg, Nature (London) 424, 817 (2003).

[10] D. Mandelik, R. Morandotti, J. S. Aitchison, and Y. Silberberg, Phys. Rev. Lett. 92, 093904 (2004).

[11] A. Trombettoni and A. Smerzi, Phys. Rev. Lett. 86, 2353 (2001); A. Smerzi and A. Trombettoni, Chaos 13, 766 (2003).

[12] B. Eiermann, T. Anker, M. Albiez, M. Taglieber, P. Treutlein, K. P. Marzlin, and M. K. Oberthaler, Phys. Rev. Lett. 92, 230401 (2004); Th. Anker, M. Albiez, R. Gati, S. Hunsmann, B. Eiermann, A. Trombettoni, and M. K. Oberthaler, ibid. 94, 020403 (2005).

[13] Yu. S. Kivshar, IEEE J. Quantum Electron. 29, 250 (1993).
[14] Yu. S. Kivshar and B. Luther-Davies, Phys. Rep. 298, 81 (1998).

[15] S. Burger, K. Bongs, S. Dettmer, W. Ertmer, K. Sengstock, A. Sanpera, G. V. Shlyapnikov, and M. Lewenstein, Phys. Rev. Lett. 83, 5198 (1999); J. Denschlag et al., Science 287, 97 (2000).

[16] L. Khaykovich, F. Schreck, G. Ferrari, T. Bourdel, J. Cubizolles, L. D. Carr, Y. Castin, and C. Salomon, Science 296, 1290 (2002); K. E. Strecker, G. B. Partridge, A. G. Truscott, and R. G. Hulet, Nature (London) 417, 150 (2002); New J. Phys. 5, 73 (2003).

[17] Yu. S. Kivshar, W. Królikowski, and O. A. Chubykalo, Phys. Rev. E 50, 5020 (1994).

[18] D. Hennig, K. Ø. Rasmussen, H. Gabriel, and A. Bülow, Phys. Rev. E 54, 5788 (1996).

[19] M. Johansson and Yu. S. Kivshar, Phys. Rev. Lett. 82, 85 (1999).

[20] V. V. Konotop and M. Salerno, Phys. Rev. E 55, 4706 (1997); V. V. Konotop and S. Takeno, ibid. 60, 1001 (1999); A. A. Sukhorukov and Yu. S. Kivshar, ibid. 65, 036609 (2002); P. G. Kevrekidis, R. Carretero-González, G. Theocharis, D. J. Frantzeskakis, and B. A. Malomed, Phys. Rev. A 68, 035602 (2003)

[21] Yu. S. Kivshar, A. R. Champneys, D. Cai, and A. R. Bishop, Phys. Rev. B 58, 5423 (1998).

[22] T. Kapitula, P. G. Kevrekidis, and B. A. Malomed, Phys. Rev. E 63, 036604 (2001); P. G. Kevrekidis, ibid. 64, 026611 (2001).

[23] D. E. Pelinovsky, P. G. Kevrekidis, and D. J. Frantzeskakis, e-print nlin.PS/0410005.

[24] Yu. S. Kivshar and M. Peyrard, Phys. Rev. A 46, 3198 (1992). 
[25] B. Sánchez-Rey and M. Johansson, Phys. Rev. E 71, 036627 (2005).

[26] T. S. Parker and L. O. Chua, Practical Numerical Algorithms for Chaotic Systems (Springer-Verlag, Berlin, 1989).

[27] S. Darmanyan, A. Kobyakov, and F. Lederer, Zh. Eksp. Teor.
Fiz. 113, 1253 (1998) [JETP 86, 682 (1998)]; P. G. Kevrekidis, A. R. Bishop, and K. Ø. Rasmussen, Phys. Rev. E 63, 036603 (2001), and references therein.

[28] M. Johansson and S. Aubry, Nonlinearity 10, 1151 (1997).

[29] S. Aubry, Physica D 103, 201 (1997). 